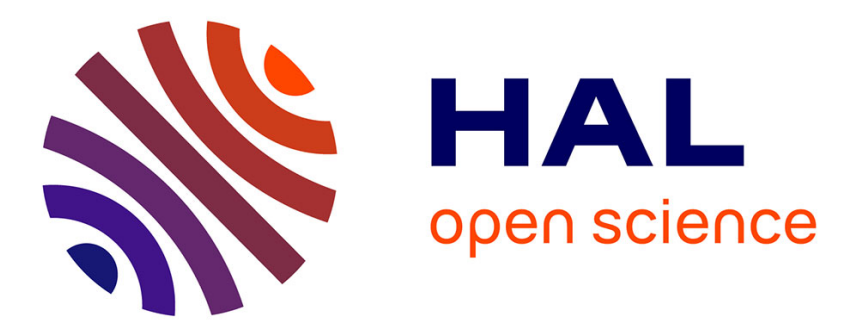

\title{
Mise au point d'une méthode d'étude de l'efficacité des produits destinés à combattre Tetranychus urticae Koch, en serre légumière
}

William Della Giustina, Jean-Baptiste Denis, Serge Meusnier, Pierre Lesaffre

\section{- To cite this version:}

William Della Giustina, Jean-Baptiste Denis, Serge Meusnier, Pierre Lesaffre. Mise au point d'une méthode d'étude de l'efficacité des produits destinés à combattre Tetranychus urticae Koch, en serre légumière. Agronomie, 1983, 3 (4), pp.307-314. hal-00884514

\section{HAL Id: hal-00884514 https://hal.science/hal-00884514}

Submitted on 1 Jan 1983

HAL is a multi-disciplinary open access archive for the deposit and dissemination of scientific research documents, whether they are published or not. The documents may come from teaching and research institutions in France or abroad, or from public or private research centers.
L'archive ouverte pluridisciplinaire HAL, est destinée au dépôt et à la diffusion de documents scientifiques de niveau recherche, publiés ou non, émanant des établissements d'enseignement et de recherche français ou étrangers, des laboratoires publics ou privés. 


\section{Mise au point d'une méthode d'étude de l'efficacité des produits destinés à combattre Tetranychus urticae Koch, en serre légumière}

William della GIUSTINA \& Jean-Baptiste DENIS $\left(^{*}\right)$

avec la collaboration technique de Serge MEuSNiER \& Pierre LeSAFrRe

I.N.R.A., Station de Zoologie,

(*) Laboratoire de Biométrie, route de Saint-Cyr - F 78000 Versailles

Cette méthode tient compte de certaines caractéristiques biologiques de l'acarien et des propriétés physicochimiques des acaricides. L'expérimentation se déroule sur plus de 40 jours afin d'observer au moins 3 cycles du ravageur. La plante-hôte est l'aubergine, variété « Bonica ». Il est procédé à une infestation artificielle sur une feuille homologue de tous les pieds, dans la serre, ce qui permet une distribution spatiale à peu près homogène. Les observations ont lieu 3 fois par semaine; elles concernent un ou deux secteurs foliaires délimités par la nervure principale et une nervure secondaire (fig. 1), en fonction de la population. On procède à 8 prélèvements par observation et par parcelle. La première application est générale quand la population sur les feuilles prélevées a atteint le seuil de traitement. Chaque parcelle est ensuite traitée indépendamment des autres, en fonction de l'évolution de sa population propre. A cet effet, un seuil d'intervention établi à 45 formes mobiles en moyenne par secteur foliaire prélevé a été défini. Lorsque ce seuil est atteint dans une parcelle, celle-ci est traitée le jour même. L'évolution de la population de chaque parcelle est représentée par une courbe qui sert à évaluer l'efficacité des produits et d'où l'on tire un certain nombre de critères, pour comparer les produits : surface sous la courbe, moyenne des dates d'application, moyenne des écarts entre les dates d'application, nombre de traitements appliqués.

Mots-clés additionnels : Cultures en serre, Lutte chimique, Acaricides, Méthode mesure efficacité produits.

\section{Method for determining the efficacy of products to control Tetranychus urticae Koch, in vegeta-} ble greenhouse.

This method takes into count some biological characteristics of the mite as well as physico-chemical properties of the acaricide. The experiment takes place over a period exceeding 40 days to observe more than 3 pest cycles. The host-plant is eggplant, « Bonica " variety. The same leaf in each plant is artificially infested in the greenhouse, thus giving a homogeneous spatial distribution. The observation of one or 2 leaf sections determined by the main and a secondary vein (fig. 1), as a function of the population, occurs 3 times a week. Two sections are observed $(1+3$ or $2+4$ as in figure 1$)$ when the postembryological population in the first section $(1$ or 2$)$ is $<50$. Only one section $(1$ or 2$)$ is observed when the postembryological population level exceeds 50. Eight samples per observation per plot. The first application is general when the population on sampled leaves has reached the treatment threshold ( 300 postembryological forms per section observed). Each plot is then treated independently, depending on the development of its own population. For this purpose, the intervention level was set for a mean population of $\mathbf{4 5}$ postembryological forms per sampled leaf section. When this level is reached in a plot, this plot is treated on the very same day. Population evolution in each plot is represented by a curve (fig. 4) used to evaluate the product efficacy. From this curve, a number of criteria are deducted to compare the different products : surface under the curve, mean of application dates, mean of intervals between application dates, number of treatments applied.

Additional key-words : Greenhouse crops, Chemical control, Acaricides, Method determination product efficacy.

\section{INTRODUCTION}

A notre connaissance, il n'existe pas dans la littérature de méthode détaillée pour étudier, en serre légumière, l'ef- ficacité pratique de produits acaricides sur le tétranyque tisserand, Tetranychus urticae Koch.

C'est la raison pour laquelle, au commencement de notre expérimentation sur ce ravageur, nous nous sommes préoc- 
cupés de la mise au point d'une telle méthode afin de permettre la comparaison précise de plusieurs produits, la détermination de doses pratiques d'emploi ainsi que la définition du rythme des applications adapté à chaque matière active. Cette méthode doit prendre en compte des facteurs primordiaux comme la brièveté du cycle biologique, la rémanence progressive des produits au fil des applications successives.

La réflexion qui nous a guidés pour sa mise au point est voisine de celle que nous avons suivie lors de l'élaboration de la méthode concernant l'aleurode des serres, Trialeurodes vaporariorum West. (della GIUSTINA \& DENIS, 1979) mais les modalités pratiques en sont cependant fort différentes : en effet, non seulement il faut tenir compte de la biologie de $T$. urticae mais aussi de sa petite taille qui, ne dépassant pas $0,5 \mathrm{~mm}$, interdit de procéder, cọmme pour l'aleurode, à un dénombrement direct et impose l'emploi d'un appareil optique au grossissement puissant.

La méthode décrite dans la présente note a déjà fait l'objet de 2 campagnes d'expérimentation dont les résultats concernant l'efficacité du fenpropathrin ont été déjà rapportés par ailleurs (della GIUSTINA et al., 1982).

\section{MÉTHODE PROPOSÉE}

\section{A. Généralités}

Pour suivre au moins 2 générations sinon 3, il convient de poursuivre l'expérimentation une quarantaine de jours après le $1^{\text {er }}$ traitement qui est appliqué à toutes les parcelles, suivant le plan d'expérience établi (cf. II.G, conduite de l'expérimentation). Chaque parcelle élémentaire est ensuite traitée indépendamment des autres, en fonction de l'évolution de sa population propre d'acariens. L'application d'un traitement s'effectue dès que la population dépasse un certain niveau que nous appelons seuil d'intervention. Une telle conduite nécessite le suivi régulier de l'évolution de la population de chaque parcelle, travail astreignant mais indispensable pour préciser correctement l'effet des produits. L'évaluation de l'efficacité des produits se fait par la comparaison des courbes d'évolution ainsi obtenues qui inclut, en particulier, le nombre d'interventions pratiquées.

\section{B. Choix de la plante-hôte}

Il serait souhaitable de pouvoir disposer d'une espèce légumière sensible seulement aux acariens. On éviterait ainsi l'interférence avec d'autres ravageurs et le risque de perturber l'expérience par l'application d'autres produits phytosanitaires. Comme une telle espèce n'existe pas, l'aubergine a été retenue pour plusieurs raisons :

- Sa feuille (unité de comptage pour l'évaluation de la population) autorise l'emploi de techniques allégeant le travail.

- L'espèce semble plus résistante aux maladies que le concombre. Le risque d'emploi de fongicides et les perturbations qui y sont liées sont ainsi diminués.

- Les populations d'acariens supportées par les feuilles d'aubergine sont importantes et ne conduisent pas à leur complet dessèchement, comme dans le cas du concombre.

Il s'agit de la variété « Bonica », conduite sur une tige, les rameaux axillaires étant étêtés, une feuille après la $1^{\mathrm{re}}$ inflorescence.
Si la notion de rentabilité n'intervient pas, il est souhaitable par souci de simplification, de ne conserver que la tige principale écimée à 1,80 ou $2 \mathrm{~m}$.

\section{Epoque de l'expérimentation}

Pour tester au mieux l'efficacité des produits, il convient que $T$. urticae se développe dans des conditions optimales. L'existence, chez cette espèce, de formes estivale et hivernale induites par l'action de plusieurs facteurs dont la durée du jour (VEERMAN, 1977) rend nécessaire la réalisation de l'expérimentation durant le printemps et le début de l'été. Dans le cas où les conditions extérieures permettent une activité de l'acarien toute l'année, il convient cependant d'éviter les périodes de jours courts pendant lesquelles sa résistance risque d'être amoindrie ou pour le moins perturbée.

\section{Préparation de la serre}

On procède à une désinfection des parois intérieures avant la plantation, afin de détruire les formes hivernantes de l'acarien. Cette pratique empêche la perturbation de l'infestation artificielle. A la dose double d'emploi $(25 \mathrm{~g} / \mathrm{hl})$, le chinométhionate se montre généralement efficace et détruit également les spores de conservation de l'oïdium (della GiUstina, 1973).

\section{E. Infestation artificielle}

Afin d'assurer une distribution homogène à la population de l'acarien lors de la $1^{\text {re }}$ application, il est nécessaire de procéder au préalable à une infestation artificielle. Trois femelles prélevées à partir d'un élevage de masse et déposées sur une feuille de chaque pied, en une seule fois, suffisent pour que le niveau d'infestation nécessaire au début de l'expérimentation soit atteint environ un mois plus tard. Il conviendra de suivre l'évolution de la population, afin de ne pas dépasser le seuil d'intervention.

Les feuilles, sur lesquelles les acariens sont déposés le même jour à la face supérieure à l'aide d'un pinceau, doivent être homologues afin de simplifier l'étude de la distribution spatiale. La numérotation des feuilles est définie par rapport à la $1^{\text {re }}$ fleur de la tige principale dont la position est relativement constante (ONILLON et al., 1976).

Les acariens doivent provenir d'un élevage utilisant la même plante-hôte. On évite ainsi le choc dû au changement de support végétal qui s'accompagne d'une mortalité importante et d'une baisse de la fécondité durant les 1res générations (JESIOTR \& SUSKI, 1976). La souche doit être sauvage, au moins pour les $1^{\text {res }}$ expérimentations, afin d'éviter l'introduction d'un paramètre trop long à mesurer : la résistance relative à une ou plusieurs familles de produits acaricides.

\section{F. Evaluation de la population}

Le but est d'estimer le plus précisément possible la population de chaque parcelle de l'expérimentation, chacune étant constituée d'un certain nombre de plantes sur les feuilles desquelles sont répartis les acariens. L'indice d'appréciation du niveau de population est le nombre moyen d'acariens sur la feuille la plus infestée de chacune des plantes.

Pour pouvoir l'estimer, il faut :

- définir la feuille la plus infestée de la plante; 
- évaluer le nombre d'acariens sur cette feuille ;

- déterminer le nombre de feuilles à prélever par observation.

Les réponses doivent se situer dans le domaine des contraintes qu'engendre l'observation binoculaire :

- un temps de comptage qui soit acceptable ;

- un volume de matériel prélevé (surface foliaire et nombre d'acariens) ne risquant pas de déséquilibrer l'évolution normale du complexe plante-ravageur.

La distinction entre les différents stades postembryonnaires est une complication inutile pour des expérimentateurs non spécialisés de sorte que les formes mobiles sont prises en compte, sans distinction.

\section{Distribution spatiale}

L'étude de la distribution spatiale des acariens sur les différentes feuilles d'une plante permet de répondre à la $1^{\text {re }}$ question mais il n'a pas été possible de formuler une règle simple comme dans le cas des aleurodes. Les investigations entreprises au cours des 2 expérimentations ont simplement fait apparaître que la feuille sur laquelle ont été déposées les femelles lors de l'infestation et ses voisines semblent représenter, au début, la strate foliaire supportant la population la plus forte.

En attendant de pouvoir établir une règle définitive pour déterminer l'étage foliaire sur lequel doivent être conduites les observations, nous recommandons de procéder à une estimation hebdomadaire. Celle-ci fixe le niveau auquel les 3 observations suivantes seront effectuées. Pour la réalisation de cette estimation, on prélève toutes les feuilles de 3 plantes réparties dans 3 parcelles différentes par condition étudiée. Ce nombre de 3 plantes n'a d'autre justification que les contraintes techniques.

On calcule leur population en pourcentage de la population totale de la plante. L'étage de la feuille qui supporte la population relative la plus élevée est choisi pour les 3 comptages hebdomadaires suivants.

Pour illustrer le choix de l'établissement de la distribution spatiale à partir de la population exprimée en pourcentage, dans l'exemple donné (tabl. 1) nous avons à choisir entre 3 étages foliaires, sur 3* plantes observées.

TABLEAU 1

Détermination de la distribution spatiale moyenne sur la plante En haut : population réelle; en bas : population évaluée en pourcentage de la population totale

Determination of the mean spatial distribution on the plant Above : actual population ; under : population evaluated as a percentage of the total population

\begin{tabular}{ccccc}
\hline \hline & $P_{1}$ & $P_{2}$ & $P_{3}$ & \\
\hline$F_{3}$ & 50 & 9 & 6 & \\
$F_{2}$ & 60 & 18 & 26 & \\
$F_{1}$ & 80 & 14 & 12 & \\
& $P_{1}$ & $P_{2}$ & $P_{3}$ & $\%$ moyen \\
\hline$F_{3}$ & 26 & 22 & 17 & 21,7 \\
$F_{2}$ & 32 & 44 & 57 & 44,3 \\
$F_{1}$ & 42 & 34 & 26 & 34 \\
\hline
\end{tabular}

$P$ : plante observée

$F$ : étage foliaire observé

$P$ : observed plant

$F$ : observed stage leaf.
D'après les résultats des comptages, c'est la feuille $n^{\circ} 2$ qui doit être prélevée bien que ce soit la feuille $n^{\circ} 1$ qui présente l'effectif d'acariens le plus important.

\section{Zone foliaire d'observation}

L'étage de la feuille dont on doit estimer la population d'acariens étant déterminé, il s'agit d'utiliser une méthode de comptage à la fois efficace et rapide qui n'altère pas trop la parcelle (population du ravageur, intégrité des plantes) par des prélèvements répétés sur les mêmes pieds. Les nervures principales et secondaires déterminent 10 secteurs à peu près constants (fig. 1). Le comptage exhaustif des 10 secteurs de 187 faces inférieures foliaires a servi de référence pour étudier diverses stratégies. La bonne symétrie des côtés gauche et droit a pu être vérifiée. Comme dans le cas des aleurodes, les distributions ont conduit à travailler sur le logarithme de l'effectif +1 , afin de stabiliser les variances des petits effectifs. Il s'avère alors que la corrélation entre les secteurs $(1+3)$ ou $(2+4)$ avec le nombre total est élevée.

Si $\mathrm{n} 1$ = nombre d'acariens sur le secteur 1

n3 = nombre d'acariens sur le secteur 3

$\mathrm{N}=$ nombre d'acariens sur une demi-feuille

Corrélation $\{\log (n 1+n 3+1), \log (N+1)\}=$ 0.925 .

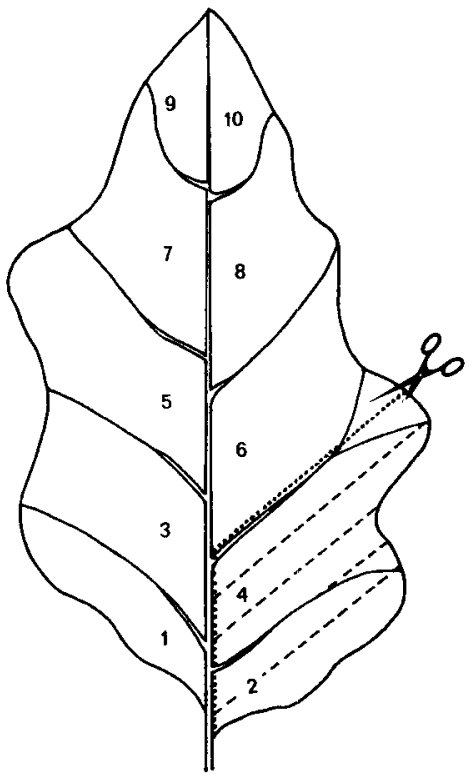

Figure 1

Face inférieure d'une feuille d'aubergine présentant les 10 secteurs identifies.

En pointillé : tracé de la découpe

En tireté : délimitation des bandes d'observation à la loupe binoculaire.

Lower face of eggplant leaf showing to 10 identified sections.

Dotted line: cutting line

Broken line : delimitation of strips for binocular observation.

La connaissance des effectifs des secteurs 1 et 3 (ou 2 et 4) permet d'expliquer 86 p. 100 de la variabilité de la population de la feuille. L'examen du graphe (fig. 2) ) $\log \left(\mathbf{n}_{1}\right.$ $\left.+n_{3}+1\right), \log (2 N+1)$ indique que plus la feuille est infestée, meilleure est cette prédiction. C'est pourquoi, dans le but de réduire encore le temps d'observation, la stratégie de comptage suivante a été simulée, à partir de l'observation du secteur 1 (ou 2).

- Si l'effectif est supérieur à une certaine limite, s'ar- 


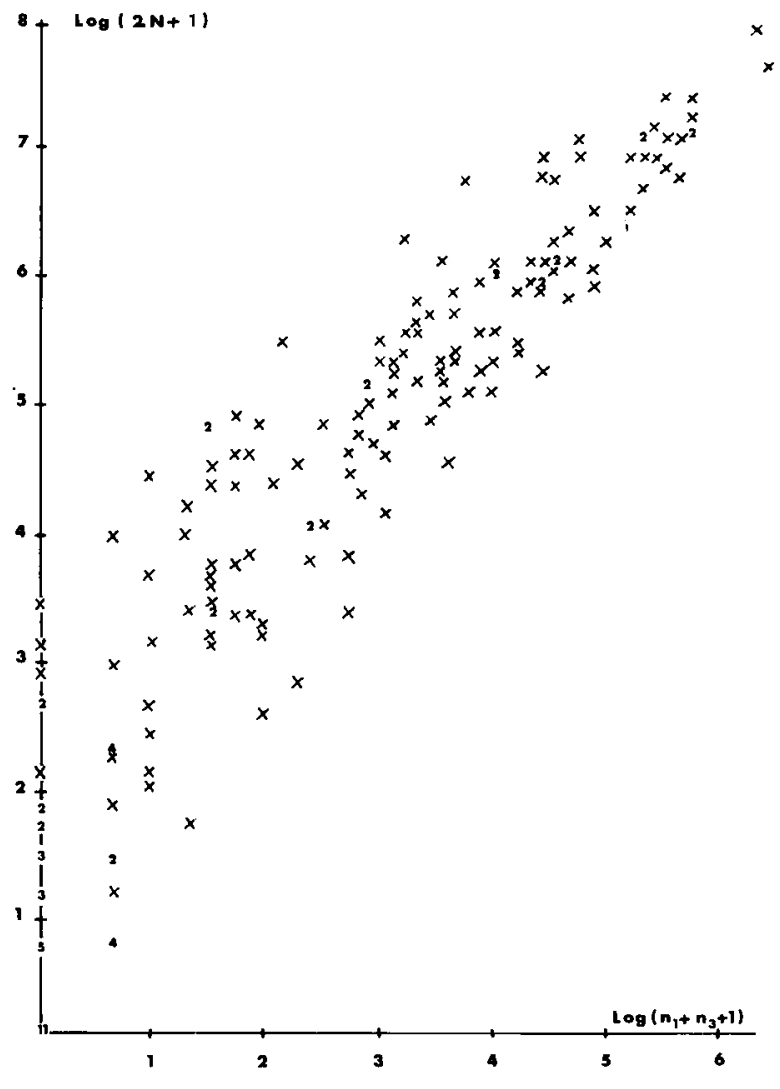

Figure 2

Relation entre le nombre d'acariens des secteurs $1+3$ et le nombre d'acariens se trouvant sur la totalité de la face inférieure de la feuille.

Un point représente une observation. Deux observations (ou plus) sur le même point sont représentées par le chiffre arabe correspondant. Il y a 11 points à l'origine.

Relationsphips between number of mites in sections $l+3$ and number of mites on the whole lower face of the leaf.

One dot represents one observation. Two (or more) observations on the same dot are represented by the corresponding number.

There are 11 dots at the origine.

rêter et estimer le nombre total d'acariens, à partir de $n_{1}$ (ou $\mathrm{n}_{2}$ ).

- Si l'effectif est inférieur à cette limite, compter aussi le secteur 3 (ou 4) et estimer le nombre total à partir de $n_{1}$ $+n_{3}$ (ou $n_{2}$ et $n_{4}$ ).

L'essai systématique des valeurs limites de 10 en 10 acariens de 0 à 290 a permis de vérifier, sur un échantillon de 437 demi-feuilles, que la limite de 50 offre la même précision statistique de prévision qu'en comptant systématiquement les secteurs 1 et 3 (ou $2+4$ ), ce qui consacre cette stratégie.

Les formules donnant l'estimation du nombre total d'acariens sont les suivantes :

$\log (N+1) \simeq 2,3+0,85 \log \left(n_{1}+1\right)$ si $n_{1}>50$

$\log (N+1) \simeq 0,9+\log \left(n_{1}+n_{3}+1\right)$ si $n_{1}<50$

$n_{2}$ et $n_{4}$ remplaçant $n_{1}, n_{3}$ si c'est la demi-feuille droite qui est échantillonnée.

Les secteurs 1 et 3 (ou 2 et 4 ) sont découpés à l'aide de ciseaux laissant en place le reste de la feuille et sont observés, immédiatement après, à la loupe binoculaire. Le grossissement doit être suffisant pour que l'on distingue correctement le $1^{\text {er }}$ stade larvaire. Il est possible, afin de favoriser l'observation de cette portion de feuille, de tracer des traits parallèles, visibles dans le champ de la loupe binoculaire. Le comptage est plus facile en faisant défiler les zones ainsi définies. L'ablation se fait en respectant la nervure principale mais en prélevant la nervure secondaire pour inclure dans le comptage les acariens qui se trouvent le long de cette dernière (fig. 3). La feuille ne semble pas présenter de lésions à la suite du prélèvement. Le choix de la demifeuille gauche ou droite se fait au hasard, par tirage au sort préalable.

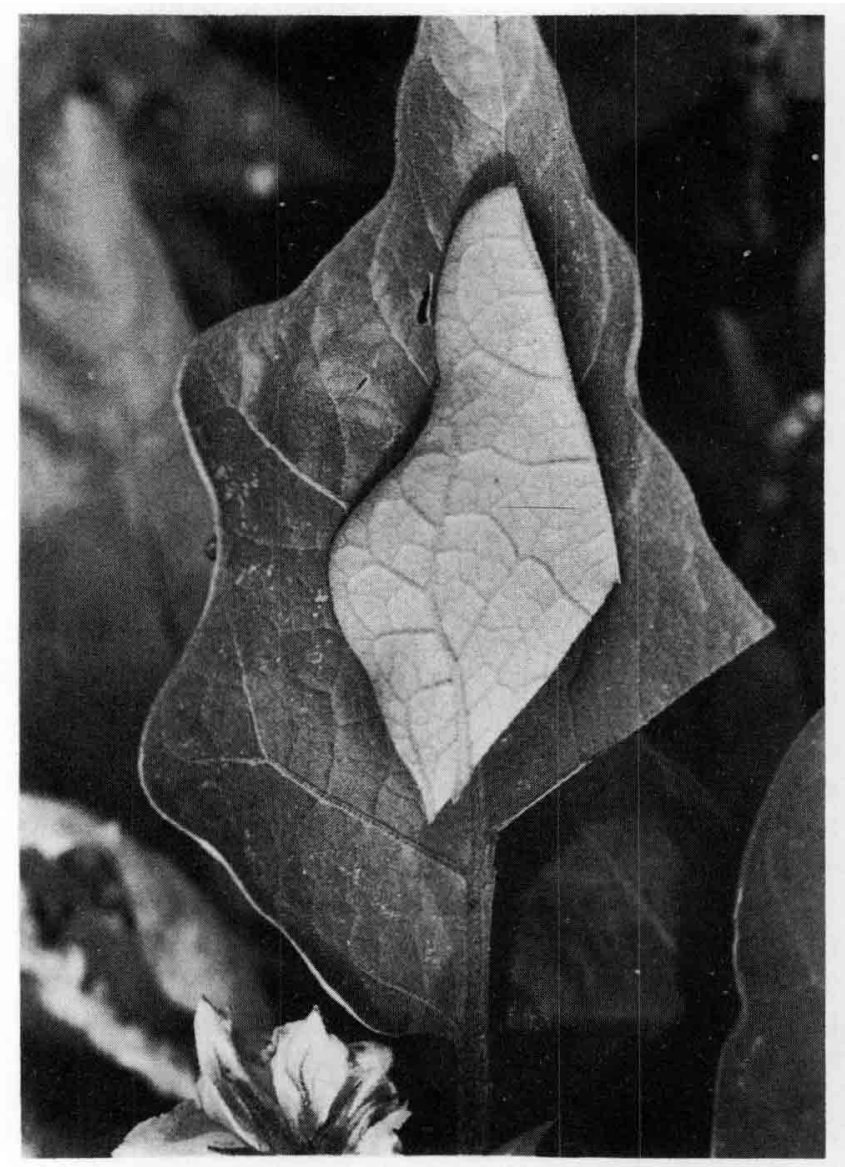

Figure 3

Rapport entre la surface foliaire prélevée aux ciseaux et celle laissée sur la plante. On reconnaît bien les secteurs 1 et 3 .

Leaf surface cut off compared with the rest of the leaf; sections I+ 3 are recognizable.

\section{Nombre de prélèvements par parcelle et par observation}

Le nombre de 8 prélèvements avait été retenu d'après les possibilités de comptage de l'équipe. L'analyse de variance du dispositif expérimental a permis, a posteriori, pour chaque date d'observation, d'estimer la variance d'échantillonnage. Le tableau 2 résume ces résultats.

\section{TABLEAU 2}

Variance d'échantillonnage obtenue par la résiduelle de l'analyse de la variance effectuée à chacune des dates Sampling variance of each observation date

\begin{tabular}{lcc}
\hline \hline & $1^{\text {re }}$ expérimentation & $2^{\mathrm{e}}$ expérimentation \\
\hline \hline date 1 & 0,96 & 2,33 \\
date 2 & 1,79 & 1,29 \\
date 3 & 1,58 & 0,28 \\
date 4 & 1,87 & 2,05 \\
date 5 & 1,99 & 1,75 \\
moyenne & 1,64 & 1,54
\end{tabular}


TABLEAU 3

Intervalles de confiance à 5 p. 100 de l'estimation de la population d'une demi-feuille calculés par l'intermédiaire de la transformée logarithmique

Confidence interval (5\%) of a half-leaf population evaluation

\section{Nombre d'acariens}

$$
\text { par } 1 / 2 \mathrm{f} \text {. }
$$

10

50

Nombre

de prélèvements

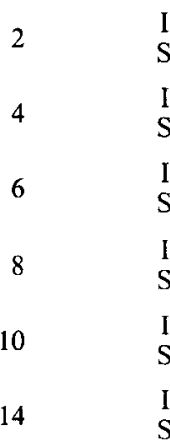

\section{1}

67

2

39

3

30

3

26

4

24

5
100

200

400

: limite inférieure, lower limit.

S : limite supérieure, upper limit.

La constance de l'ordre de grandeur de ces estimations est à noter : il est possible, de ce fait, de calculer théoriquement les intervalles de confiance à $5 \mathrm{p} .100$ pour différents nombres de plantes échantillonnées, sur la transformée logarithmique (tabl. 3), en utilisant la valeur 1,65 supérieure aux 2 moyennes. Remarquons que cet intervalle de confiance dépend du niveau de population du fait de la transformation logarithmique.

Il est évident que plus le nombre de feuilles échantillonnées est important, plus l'intervalle de confiance est restreint. Mais la réduction de ce dernier n'est pas proportionnelle au nombre d'échantillons alors que le temps de comptage l'est exactement.

Ces valeurs amènent à proposer que le nombre de feuilles observées soit 8. Dans le cas de possibilités techniques supérieures, il est préférable d'augmenter le nombre de blocs plutôt que le nombre d'observations dans une même parcelle. Les pieds sur lesquels les feuilles sont prélevées sont tirés au sort.

\section{G. Conduite de l'expérimentation}

Un dispositif comprenant au moins 4 blocs complets (5 dans le cas où il n'y a que 2 traitements) est préconisé. L'infestation initiale donnant une répartition suffisamment homogène, les blocs seront disposés de manière classique. La totalité de la serre est réservée à l'expérimentation, sans parcelle témoin non traitée qui servirait de foyer d'infestation. Chaque parcelle est séparée de ses voisines par des cloisons translucides, afin d'éviter toute projection de produit au moment des traitements. Il est souhaitable qu'une parcelle comprenne au moins 60 pieds. Les comptages se font 3 fois par semaine (lundi, mercredi, vendredi par exemple). L'échantillonnage s'effectue sur l'ensemble de la parcelle. La $1^{\text {re }}$ application, généralisée à toutes les parcelles, s'effectue lorsque le nombre moyen de formes mobiles par demi-feuille dépasse $740,\left(n_{1}+n_{3}>300\right)$, ce qui correspond à $\log (\mathrm{N}+1)>6,6$.
Les traitements suivants ont lieu indépendamment dans chacune des parcelles lorsque le nombre moyen observé par demi-feuille dépasse 110 , soit $n_{1}+n_{3}>45$, ce qui correspond à $\log (N+1)>4,73$.

Il convient de préciser que les moyennes s'effectuent sur les logarithmes comme le montre l'exemple du tableau 4.

Le seuil d'intervention de 4,73 ne s'applique que lorsque la courbe représentant l'évolution de la population est en phase ascendante.

Les individus moribonds sont comptés comme vivants.

Les applications doivent être soignées. Les acaricides autorisés en culture légumière agissent surtout par contact, aussi est-il nécessaire de bien mouiller les 2 faces de la feuille, ce qui implique une quantité de bouillie importante ( $>3000$ $1 / \mathrm{ha}$, en pleine végétation).

Il est conseillé aussi de noter les heures d'application des

TABLEAU 4

Exemple de comptage dans une parcelle

Example of population counting in a plot

\begin{tabular}{cccc}
\hline $\begin{array}{c}\mathbf{N}^{\circ} \text { du } \\
\text { comptage }\end{array}$ & $\begin{array}{c}\text { Comptage } \\
\mathbf{n}_{1}\left(\text { ou } \mathrm{n}_{2}\right)\end{array}$ & $\begin{array}{c}\text { Comptage } \\
\left.\mathrm{n}_{3} \text { ou } \mathbf{n}_{4}\right)\end{array}$ & $\begin{array}{c}\text { Estimation de } \\
\text { Log }(\mathbf{N}+1)\end{array}$ \\
\hline 1 & 54 & - & 5,71 \\
2 & 12 & 5 & 3,79 \\
3 & 30 & 22 & 4,87 \\
4 & 5 & 17 & 4,04 \\
5 & 57 & - & 5,75 \\
6 & 62 & - & 5,82 \\
7 & 9 & 19 & 4,27 \\
8 & 39 & 42 & 5,31 \\
\hline
\end{tabular}

Moyenne des $\log (N+1)=4,95>4,73=$ la parcelle est traitée. Mean $\log (\mathrm{N}+1)=4,95>4,73=$ the plot is treated. 
acaricides car les arthropodes comme les mammifères présentent des réponses variables suivant le rythme circadien de leurs activités biologiques (et aussi probablement en fonction d'autres cycles plus longs). Il s'ensuit, en particulier pour la mesure de l'effet choc ou celle de l'efficacité de produits à action fugace, que des résultats éloignés peuvent s'expliquer simplement par ce phénomène encore mal étudié chez les insectes et acariens qu'est la chronopharmacologie ou chronotoxicité.

\section{H. Analyse statistique}

La démarche est la même que dans le cas des aleurodes. Il faut comparer p.b courbes (où $p$ est le nombre de produits testés, b le nombre de blocs) qui résument chacune le comportement d'une parcelle. Pour se ramener à la comparaison de valeurs numériques, chaque courbe est caractérisée par un certain nombre de critères sur lesquels une analyse de la variance correspondant au plan d'expérience est pratiquée.

Les critères et leur interprétation sont :

$\mathrm{Cl}$ : surface sous la courbe qui traduit l'effet global du protocole suivi au cours de l'expérimentation.

C2 : moyenne des dates d'application. La date de l'application généralisée étant prise nulle par convention. Ce critère mesure la rémanence du produit car, toutes choses égales par ailleurs, plus tôt il a été administré, plus le critère est faible.

C3 : moyenne des écarts (en jours) entre les applications. Il mesure aussi la persistance d'action du produit mais élimine la durée d'efficacité de la dernière application que l'on ne connaît pas puisque l'expérimentation a été arrêtée avant.

C4 : nombre de traitements appliqués sur toute l'expérimentation. Il mesure la persistance d'action du produit d'un point de vue économique. Pour ce dernier critère (le nombre de traitements étant de quelques unités), la pratique de l'analyse de variance n'est pas recommandable. Il est préférable de procéder à une analyse statistique non paramétrique basée sur les rangs dont on trouvera le détail, par exemple, dans HoLlANDER \& WOLFE (1973).
TABLEAU 5

Exemple de données servant au calcul des différents critères d'une parcelle, lors d'une expérimentation

Example of data used to evaluate criteria of one plot, during the experiment

\begin{tabular}{|c|c|c|c|}
\hline $\mathrm{t}$ & $\log (N+1)$ & $\mathbf{N}$ & Pondération \\
\hline (0) & 7,346 & 1549 & 1 \\
\hline 2 & 6,173 & 479 & 2,5 \\
\hline 5 & 5,969 & 390 & 2,5 \\
\hline 7 & 5,636 & 279 & 2 \\
\hline 9 & 4,564 & 95 & 2,5 \\
\hline 12 & 4,638 & 102 & 2,5 \\
\hline (14) & 4,851 & 127 & 2 \\
\hline 16 & 3,553 & 34 & 3,5 \\
\hline 21 & 3,052 & 20 & 3,5 \\
\hline 23 & 2,832 & 16 & 2,5 \\
\hline 26 & 2,208 & 8 & 2,5 \\
\hline 28 & 2,846 & 16 & 2 \\
\hline 30 & 3,028 & 20 & 2,5 \\
\hline 33 & 3,289 & 26 & 2,5 \\
\hline 35 & 4,035 & 56 & 2 \\
\hline 37 & 3,790 & 43 & 2,5 \\
\hline (40) & 4,750 & 115 & 2,5 \\
\hline 42 & 2,708 & 14 & 2 \\
\hline 44 & 3,193 & 23 & 1 \\
\hline
\end{tabular}

$\mathrm{t}=$ numéro des jours de comptage.

Les dates entre parenthèses correspondent à celles où un traitement de la parcelle a été effectué.

$\mathbf{t}=$ number of counting days

Days between parenthesis = days when plot was treated.

A titre d'illustration, la valeur des différents critères sera calculée à partir des observations (tabl. 5) effectuées dans une parcelle.

La figure 4 donne la courbe d'évolution qui lui corres-

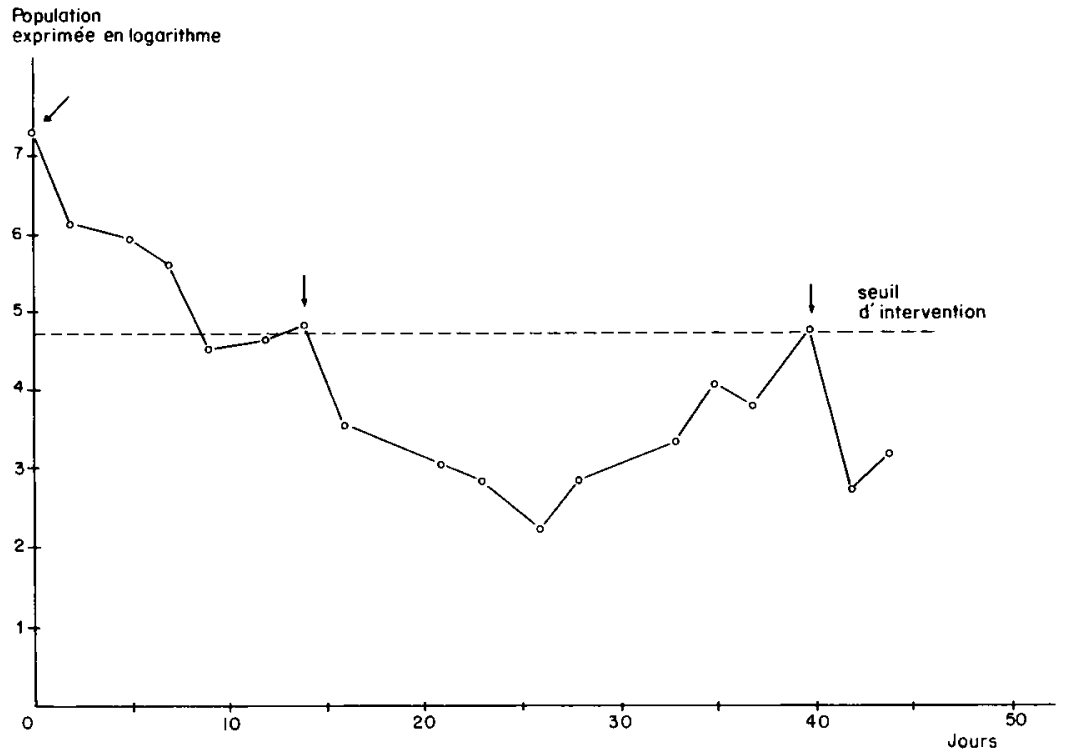

Figure 4

Evolution de la population d'une parcelle. Les flèches indiquent qu'un traitement a eu lieu. L'utilisation directe de la transformée logarithmique évite d'employer le papier semi-logarithmique.

Population evolution in one plot. Arrows indicate occurrence of a treatment. 
pond. $\mathrm{C} 1$, la surface sous la courbe, se calcule par décomposition en trapèzes rectangles, ce qui revient à faire la somme des valeurs pondérées par la moyenne des écarts entre les jours d'observation qui entourent la date. Exemple, pour $\mathrm{t}=9$ la pondération est :

$$
\frac{(9-7)+(12-9)}{2}=2.5
$$

Pour $\mathrm{t}=0$, c'est simplement $\frac{(2-0)}{2}=1$. Ces pondérations sont données pour chaque date dans le tableau 5. La somme de ces pondérations est égale au nombre de jours de l'expérimentation, ce qui permet d'opérer une vérification simple. Dans cet exemple, le calcul donne :

$$
\mathrm{C} 1=176,911
$$

Les autres critères se calculent immédiatement :

$$
\begin{aligned}
& \mathrm{C} 2=\frac{0+14+40}{3}=18 \\
& \mathrm{C} 3=\frac{(14-0)+(40-14)}{2}=20 \\
& \mathrm{C} 4=3
\end{aligned}
$$

\section{Tableau récapitulatif des principales opérations}

Afin de faciliter la compréhension de la méthode, le tableau 6 présente la chronologie des différents travaux pratiques et techniques ainsi que les principales informations quantitatives qui y sont liées.

Il est clair qu'avant le traitement généralisé (jour 0) les dates qui sont indiquées le sont à titre indicatif.

\section{DISCUSSION ET CONCLUSION}

La méthode proposée a été élaborée au cours de 2 expérimentations menées en 1981 concernant le fenpropathrin. Sa conception procède de la même réflexion que celle qui a conduit à la réalisation de la méthode d'étude de l'efficacité des insecticides destinés à combattre l'aleurode des serres, en 1979. Il s'agit de prolonger l'expérimentation pendant une période suffisamment longue, avec des observations fréquentes, afin de faire ressortir les qualités des produits étudiés. Cette méthode permet aussi de concevoir une stratégie de lutte en définissant non seulement la dose pratique d'emploi mais également le rythme des applications en fonction des qualités de chaque produit, à partir d'un seuil d'intervention. La définition d'un tel seuil permet de montrer le bien-fondé d'applications en nombre modéré (WYMAN et al., 1979). De nombreux problèmes ont été résolus : dispositif expérimental, dimension de la parcelle, seuils d'intervention, rythme des observations, secteurs foliaires à observer liés au niveau de population, nombre de prélèvements par parcelles... Il faut noter à cet effet la règle simple proposée pour le choix de la surface foliaire à prélever. Il reste cependant à confirmer ces résultats par une $2^{\mathrm{e}}$ année d'expérimentation.

Certaines questions méritent encore d'être approfondies. Il serait souhaitable de résoudre le problème de la distribution spatiale par une règle simple (si elle existe) afin d'éviter des comptages hebdomadaires supplémentaires alors que ceux que l'expérimentation proprement dite nécessite sont amplement suffisants. Il serait intéressant également d'essayer de mettre au point une méthode plus rapide de prélèvement des acariens, qui permette d'analyser simultanément l'ensemble des secteurs foliaires d'une même parcelle. L'em-

TABLEAU 6

Tableau récapitulatif des principales opérations nécessitées par la méthode

Time-table of the main operations used during the method

$\mathrm{N}^{\circ} \mathrm{du}$ jour Opérations

- 60 Désinfection des parois intérieures, la serre étant vide.

- 55 Plantation des aubergines. 4 ou 5 blocs, 60 pieds par parcelle.

- 30 Infestation artificielle : 3 q par pied, sur la même feuille. On surveillera le développement de la population jusqu'à - 8 .

- 8 Première estimation du niveau de la population: distribution des acariens sur 3 plantes par traitement.

- 3 Seconde estimation de même type que la précédente. Décision de la date du traitement généralisé, choix de la feuille à prélever, tirage au sort des parcelles.

- 1 Installation des cloisons translucides séparant les parcelles.

0 Détermination de la population de chaque parcelle par analyse de 8 fragments foliaires comprenant 2 Secteurs chacun. Le nombre de secteurs observés à chaque fois dépend de l'importance de la population qui s'y trouve. Première et seule application pour laquelle toutes les parcelles sont traitées le même jour.

1 Eventuellement, pour la mesure de l'effet choc.

2

$4\}$

7 )

8

9

11

14

Comptages de la semaine $n^{\circ} 1$.

Choix de l'étage foliaire pour la semaine $\mathrm{n}^{\circ} 2$ à partir de l'observation de toutes les feuilles de 3 plantes par traitement.

Comptages de la semaine $n^{\circ} 2$

Ainsi de suite jusqu'à la fin de l'expérience.

Le produit est appliqué dans une parcelle quand la population d'acariens atteint lé seuil d'intervention.

Les travaux culturaux sont réalisés au moment opportun. 
ploi d'une brosse adaptée à l'épiderme des feuilles d'aubergine semble du domaine du possible (PETERS \& BERRY, 1980) mais il ne faudrait pas que l'avantage du gain de temps soit obtenu au détriment de l'efficacité du prélèvement. En effet, les récoltes d'individus dans la glu ou l'alcool ne permettent plus de distinguer les spécimens déjà morts de ceux qui étaient encore vivants au moment de leur ramassage.

La méthode proposée, malgré les astreintes dues essentiellement à la petite taille du ravageur, permet une approche rigoureuse de l'efficacité des acaricides contre $T$. urticae ainsi que la définition d'un rythme des traitements au long d'une culture, si l'on tient compte également de la chronopharmacologie qui peut expliquer des différences d'ef- ficacité quand les produits sont appliqués à des heures variables dans la journée.

Reçu le 9 juillet 1982.

Accepté le 24 novembre 1982.

\section{REMERCIEMENTS}

Nous tenons à remercier Mme Pralavorio et M. Onillon d'avoir bien voulu lire le manuscrit et apporter leurs remarques et corrections. Nos remerciements vont également à M. CALviÈre, technicien de la Sté Agrishell qui a utilisé cette méthode et nous a fait part de ses commentaires.

\section{RÉFÉRENCES BIBLIOGRAPHIQUES}

della Giustina W., 1973. Epidémiologie des ravageurs aériens des cultures légumières en serre. Moyens nouveaux de lutte. Déf. Vég., 163, 250-257.

della Giustina W., Denis J.-B., 1979. Méthode d'étude de l'efficacité des produits destinés à combattre Trialeurodes vaporariorum West., en serre expérimentale. Ann. Zool. Ecol. anim., 11, 259-270.

della Giustina W., Lesaffre P., Meusnier S., Lereec A., 1982. Etude d'un nouveau pyrethrinoïde, le fenpropathrin, vis-à-vis de $T$. urticae Koch en serre. Méthode utilisée et résultats obtenus. Phytiatr. Phytopharm. (sous presse).

Hollander N., Wolfe D.A., 1973. Non parametric statistical methods. John Wiley and Sons - New York, 503 p.

Jesiotr L.J., Suski Z.W., 1976. The influence of the host-plants on the reproduction potential of the two-spotted species. Ekol. pol., 24, 407-411.
Onillon J.-C., Onillon J., Di Pietro J.-P., 1976. Résultats préliminaires du contrôle biologique de l'aleurode des serres, $T$. vaporariorum West. (Homopt. Aleurodidae) par E. formosa G. (Hymenopt. Aphelinidae) en serre d'aubergine. Bull. O.I.L.B. S.R.O.P. "Lutte intégrée en culture sous serre "1976, 4, 138-150.

Peters K.M., Berry R.E., 1980. Resistance of Hop varieties to twospotted spider mite. J. econ. Entomol. 73, 230-234.

Veerman A., 1977. Aspect of the induction of diapause in a laboratory strain of the mite Tetranychus urticae. J. Insect Physiol., 23, 703-711.

Wyman J.A., Oatman E.R., Voth V., 1979. Effects of varying twospotted spider mite infestation levels on strawberry yield. J. econ. Entomol., 72, 747-753. 